INPLASY

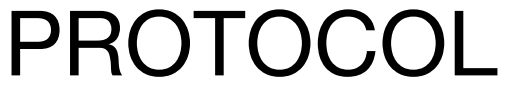

To cite: Ilami et al. Application of immersive technology interventions in sexual victims: A systematic review protocol. Inplasy protocol 202190081. doi:

10.37766/inplasy2021.9.0081

Received: 23 September 2021

Published: 23 September 2021

Corresponding author:

Ashkan Ilami

ashkan.ilami@graduate.curtin.edu.au

Author Affiliation:

1) Kish International Campus, Tehran University of Medical Sciences,

Tehran, Iran; 2) Students' Scientific

Research Center (SSRC), Exceptional Talents Development Center (ETDC),

Tehran University of Medical

Sciences (TUMS), Tehran.

Support: The study is selffunded.

Review Stage at time of this submission: Data extraction.

Conflicts of interest:

None declared.

\section{Application of immersive technology interventions in sexual victims: $A$ systematic review protocol}

Ilami, A; Amini-Tehrani, M; Zamanian, H.4.

Review question / Objective: The aim of this systematic review is to critically evaluate the current evidence regarding the immersive technology interventions in sexual victims in terms of applied methodology and patient-related outcomes. Accordingly, this systematic review will address the following questions: 1) what are the general methodological features (study design, sample and related variables, intervention content and related variables, so forth) of immersive technology interventions targeting sexually victimized individuals, 2) to what extent immersive technology interventions targeting sexually victimized individuals are promising in terms of methodological outcomes (such as feasibility, safety, so forth), and 3) to what extent the immersive technology interventions have been effective in addressing biopsychosocial outcomes in sexually victimized individuals.

INPLASY registration number: This protocol was registered with the International Platform of Registered Systematic Review and Meta-Analysis Protocols (INPLASY) on 23 September 2021 and was last updated on 23 September 2021 (registration number INPLASY202190081).

\section{INTRODUCTION}

Review question / Objective: The aim of this systematic review is to critically evaluate the current evidence regarding the immersive technology interventions in sexual victims in terms of applied methodology and patient-related outcomes. Accordingly, this systematic review will address the following questions: 1) what are the general methodological features (study design, sample and related variables, intervention content and related variables, so forth) of immersive 
technology interventions targeting sexually victimized individuals, 2) to what extent immersive technology interventions targeting sexually victimized individuals are promising in terms of methodological outcomes (such as feasibility, safety, so forth), and 3) to what extent the immersive technology interventions have been effective in addressing biopsychosocial outcomes in sexually victimized individuals.

Condition being studied: Sexual victimization is considered as a type of traumatic experience that may contribute to, if not independently cause, a range of negative short-term and long-term biopsychosocial consequences such as panic attacks, post-traumatic stress symptomatology, social isolation, depression, and suicidal ideation and attempts. The prevalence of sexual victimization has been documented mainly in the developed countries, while it is considered to be underreported as a result of perceived insecurities and threats. Sexual victimization survivors may include a diverse population, including but not limited to children and adolescents, adults, people with disabilities, sex workers, college students, workforces, sexual minorities, military personnel, combatants, and veterans. Sexual victimization may also occur as a type of domestic or intimate partner violence as well. For sexual victimization may occur in various settings and types, we have adopted this notion to cover a range of related issues, including sexual violence, sexual assault, sexual harassment, sexual abuse, sexual trauma, and sexual coercion. In our applied terminology, victimization of any actual or threatening unwanted sexual advances/ encounters/experiences that cause perceived distress and/or physical harm was considered as a defining feature of the core concept of sexual victimization.

\section{METHODS}

Participant or population: Participants are sexual victimized individuals either confirmed/reported by health systems (e.g.; medical reports - psychiatric reports); or legal systems (e.g.; police reports - judge reports); or just self-reported by the victims/survivors with no limitation for age, gender, sexual orientation, ethnicity, lifestyle, socioeconomic status, or clinical characteristics.

Intervention: The intervention is based upon any immersive technologies, including augmented reality, virtual reality, mixed reality. There is no restriction in terms of intervention mode, and all preventive, assessment, therapeutic, informational, or educational, so forth, interventions will be included.

Comparator: Although having control group is not an inclusion criterion in this systematic review, all applied comparators in eligible studies will be reported and included in data synthesis to inform further investigations.

Study designs to be included: Studies using quasi-experimental design will be included if they report at least one active arm of eligible participants (i.e., sexually victimized individuals), who have received immersive technology interventions (experimental group). Studies with and without control groups and comparators could be included. There will be no restrictions concerning control groups/ comparators and follow-up time periods.

Eligibility criteria: Further inclusion decisions will be based on the following criteria: Outcomes: Studies objectively evaluated and reported at least one outcome (either methodological or biopsychosocial outcomes) after the intervention. Setting: There is no specific limitation for care settings where the studies were conducted and all healthcare settings such as hospitals, care centres, sexual abuse/assault centres, rehabilitation centres, nursing homes, and communitybased care settings, as well as incarceration centres can be included in this study. Language: Only English language articles were included. Criteria for exclusion included systematic reviews, reviews, theses, dissertations, book chapters, letters, editorials, conference papers, articles not published in a peer- 
reviewed journal, articles that used participants who were not sexual victims, case studies, and studies published in languages other than English, and articles that their full-texts were not available or accessible.

Information sources: Major databases of Medline/Pubmed, Scopus, Web of Science core collection databases (including $\mathrm{SSCI}$, SCIE, AHCI, ESCI), and PsycINFO are considered as information sources to find eligible publications. Resources that are not currently peer-reviewed and published will not be included to reduce the bias regarding methodological evaluations. Therefore, dissertations, grey literature, and trial registries will not be evaluated.

Main outcome(s): We have categorized the measured outcomes into two groups: "methodological outcomes" and "biopsychosocial outcomes." The methodological outcomes are defined as those methodological considerations that have been taken as the primary study outcomes (or one of the study outcomes), which may include the feasibility, acceptability (i.e., participant engagement), user friendly (for service users or clinicians), usefulness, validity, relevance, safety, so forth. The biopsychosocial outcomes are defined as the patientrelated outcomes that have been taken as the primary study outcomes (or one of the study outcomes), including any physiological, psychological, or social indicators of mental or physical health. All outcomes should be assessed in post-test mode at least.

Data management: Data will be handled using EndNote application software in merging the results from the resources and finding duplications. Afterwards, the Rayyan system (https://rayyan.ai/) will be employed to screen the dataset according to their titles and abstracts and irrelevant or entirely off-topic articles will be excluded. If an abstract will be considered relevant or ambiguous, the full text will be reviewed to apply the inclusion and exclusion criteria. So afterwards, full texts of the remaining articles will be assessed for eligibility and will be excluded with reasons and candidate studies will be again evaluated against inclusion/exclusion criteria for final inclusion. Two reviewers will independently identify the finally included articles and disagreements will be resolved by a round of discussion with the presence of the third co-author as the supervisor of the study. Regarding data extraction, two independent authors will extract the following information from included articles using predefined data fields which include three main aspects of the selected articles: 1) General methodological features, such as authors, settings, study design, statistics, sample and related variables, intervention content and related variables, applied measures, so forth, 2) methodological outcomes, 3) biopsychosocial outcomes.

Quality assessment / Risk of bias analysis: The quality of included publications was assessed using the Modified JADAD scale. The early version of the 3-item JADAD scale measures the quality of clinical trials regarding three aspects of randomization, double-blindness, description of withdrawal and dropouts. In addition to these three factors, the modified version includes a clear description of inclusion/ exclusion criteria, assessment of adverse effects, and statistical analysis. Each criterion would be scored using a yes/no question that would yield either 1,0 or -1 score; thus, the total score would be in the range of 0 to 8 .

Strategy of data synthesis: Narrative synthesis is adopted to synthesize the extracted data. This strategy will allow us to capture various dimensions of the application of immersive technology interventions in sexual victims and address the study aims appropriately. Therefore, the synthesized results will be organized based on major aspects including a description of the general methodological features of eligible publications, quality assessment and risk of bias, and evaluation of two broad categories of outcomes, including methodological outcomes and biopsychosocial outcomes. If applicable, the populations of interests will be 
categorized to report specific results; otherwise, the included participants will be described using their gender, age, nationality, ethnicity, and related feature. Meta-analysis will be conducted on at least two almost homogenous publications with moderate to high quality, which report the effectiveness of a consistent intervention on any set of similar biopsychological outcomes. The meta-analysis method will be decided based on the nature of data and according to the best practice, which cannot be specified before data extraction and quality assessment phases in this review study. The synthesis will address the limitations and recommendations that may further the application of immersive technologies among sexual victims.

Subgroup analysis: If meta-analysis is applicable and enough articles were available in each subgroup, the subgroup analysis across gender groups will be conducted to determine whether there is a gender effect in the effectiveness of the immersive technology interventions in sexual victims.

Sensitivity analysis: If meta-analysis was applicable in the dataset, sensitivity analysis would be conducted according to the nature of data and best practices in the field.

Language: Only English language publications will be included.

Country(ies) involved: IRAN.

Keywords: Immersive technologies; Augmented reality; Virtual reality; Sexual violence; Sexual assault; Sexual abuse; Sexual trauma; Sexual coercion; Sexual victims; Intervention.

Dissemination plans: The final report will be disseminated through peer-review journals primarily engaged in research on interpersonal violence, preferably with a focus on sexual victimization.

Contributions of each author:

Author 1 - Ashkan Ilami Conceptualization, Study design, administration, data screening, initial draft, synthesis.

Email: ashkan.ilami@graduate.curtin.edu.au Author 2 - Mohammadali Amini-Tehrani data screening, initial draft, synthesis.

Email: amini.m.ali@ut.ac.ir

Author 3 - Hadi Zamanian - Study design, methodology, synthesis, critical review, supervision.

Email: zamanian@alumnus.tums.ac.ir 